\title{
NONCOMMUTING UNITARY GROUPS AND LOCAL BOUNDEDNESS
}

\author{
JAN RUSINEK
}

\begin{abstract}
We exhibit two unitary strongly continuous one-parameter groups $\left(e^{A_{1} t}\right)_{t \in \mathbf{R}}$ and $\left(e^{A_{2} t}\right)_{t \in \mathbf{R}}$ acting in a Hilbert space $H$, a dense subspace $D$ of $H$ contained in the domains of $A_{1}$ and $A_{2}$ such that $\left(A_{1}(D) \cup A_{2}(D)\right) \subset D$ and $\left(e^{A_{1} t}(D) \cup e^{A_{2} t}(D)\right) \subset D$ for each $t \in \mathbf{R}$, and an element $x$ of $D$ such that the function $t \rightarrow\left\|A_{1} e^{A_{2} t} x\right\|$ is not locally bounded.
\end{abstract}

1. Introduction. Let $H$ be a Hilbert space. Suppose that $A_{1}$ and $A_{2}$ are the generators of unitary strongly continuous one-parameter groups $\left(e^{A_{1} t}\right)_{t \in \mathbf{R}}$ and $\left(e^{A_{2} t}\right)_{t \in \mathbf{R}}$, respectively, acting in $H$. Suppose, moreover, that $D$ is a dense subspace of $H$ contained in the domains of $A_{1}$ and $A_{2}$ such that $\left(A_{1}(D) \cup A_{2}(D)\right) \subset D$ and $\left(e^{A_{1} t}(D) \cup e^{A_{2} t}(D)\right) \subset D$ for each $t \in \mathbf{R}$. The following is an open problem: Does $A_{1} A_{2} x=A_{2} A_{1} x$ for all $x \in D$ imply that the groups $\left(e^{A_{1} t}\right)_{t \in \mathbf{R}}$ and $\left(e^{A_{2} t}\right)_{t \in \mathbf{R}}$ commute? This is a particular case of a more general question about integrability of Lie algebra representations. Under the additional assumption:

for each $x \in D$, the function $t \rightarrow\left\|A_{2} e^{A_{2} t} x\right\|$ is locally bounded,

the problem above is solved in the affirmative. More generally, known results on integration of Lie algebra representations involve in a crucial way conditions analogous to $(*)$ (cf. [1, Theorems 3.4 and 9.1]). In this connection, P. E. T. Jørgensen and R. T. Moore (cf. [1, Remark, p. 67]) raised the question whether for $A_{1}, A_{2}, D$ as above, condition (*) is automatically fulfilled. We show that in general this is not the case. Our result shows, among other things, that the topological assumption on the domain $D$ in Proposition 3.6 of [1] is essential.

2. The result. Given a subset $A$ of $\mathbf{R}$ and $x \in \mathbf{R}$, let $A+x=\{y \in \mathbf{R}: y=a+x$, $a \in A\}$.

For each integer $n \geqslant 2$, let $I_{n}=\left(2^{n}, 2^{n}+3^{-n}\right)$ and $J_{n}=\left(2^{n}+2 \cdot 3^{-n}, 2^{n}+\right.$ $\left.3^{-n+1}\right)$.

Proposition 2.1. Given $x, y \in \mathbf{R}$, there exists at most one pair of integers $m \geqslant 2$, $n \geqslant 2$ such that

$$
\left(I_{n}+x\right) \cap\left(J_{m}+y\right) \neq \varnothing .
$$

Received by the editors March 6, 1986 and, in revised form, July 3, 1986.

1980 Mathematics Subject Classification (1985 Revision). Primary 47D10. 
Proof. Suppose that for $x, y \in \mathbf{R}$ and integers $m_{i} \geqslant 2, n_{i} \geqslant 2(i=1,2)$, the sets $\left(I_{n_{i}}+x\right) \cap\left(J_{m_{i}}+y\right)$ are not empty. Then

$$
y-x=2^{m_{i}}-2^{n_{i}}+\varepsilon_{i}-\eta_{i}
$$

where $2 \cdot 3^{-m_{i}}<\varepsilon_{i}<3^{-m_{i}+1}$ and $0<\eta_{i}<3^{-n_{i}}$. Since $\left|\varepsilon_{1}-\eta_{1}\right|+\left|\varepsilon_{2}-\eta_{2}\right|<4 / 9$ $<1$, it follows that

$$
2^{m_{1}}-2^{n_{1}}=2^{m_{2}}-2^{n_{2}}
$$

and

$$
\varepsilon_{1}-\eta_{1}=\varepsilon_{2}-\eta_{2}
$$

Equation (2.1) implies that either $m_{1}=m_{2}$ and $n_{1}=n_{2}$, or $m_{1}=n_{1}$ and $m_{2}=n_{2}$. In the latter case, we have $3^{-m_{i}}<\varepsilon_{i}-\eta_{i}<3^{-m_{i}+1}$, whence, by (2.2), $m_{1}=m_{2}=$ $n_{1}=n_{2}$. Thus, in both cases, $m_{1}=m_{2}$ and $n_{1}=n_{2}$.

The proof is complete.

Let $I=\bigcup_{n=2}^{\infty} I_{n}$ and $J=\bigcup_{n=2}^{\infty} J_{n}$. As an immediate corollary, we obtain

Proposition 2.2. For each $x, y \in \mathbf{R}$, the set $(I+x) \cap(J+y)$ is bounded.

For each $n \in \mathbf{N}$, let $C^{n}(\mathbf{R})$ be the space of all complex functions on $\mathbf{R}$ which possess continuous derivatives of order $\leqslant n$. Let $C^{\infty}(\mathbf{R})=\bigcap_{n=1}^{\infty} C^{n}(\mathbf{R})$. For $n \in \mathbf{N}$ $\cup\{\infty\}$, let $C_{0}^{n}(\mathbf{R})$ be the space of functions in $C^{n}(\mathbf{R})$ with compact support. For any function $f$ on $\mathbf{R}$, we denote by $\operatorname{supp} f$ the support of $f$.

For each integer $n \geqslant 2$, let $\varphi_{n}$ be a nonnegative function in $C_{0}^{\infty}(\mathbf{R})$ with support in $I_{n}$, such that $\left|\varphi_{n}^{(k)}\right| \leqslant 1$ for $k \leqslant n$. Set

$$
\varphi(x)=\sum_{n=2}^{\infty} \varphi_{n}(x) \quad(x \in \mathbf{R}) .
$$

Clearly, supp $\varphi \subset I$. Moreover, all derivatives of $\varphi$ are square integrable, since for any $k \in \mathbf{N}$,

$$
\int_{\mathbf{R}}\left(\varphi^{(k)}(x)\right)^{2} d x \leqslant \sum_{n=2}^{k} \int_{\mathbf{R}}\left(\varphi_{n}^{(k)}(x)\right)^{2} d x+\sum_{n=k+1}^{\infty} 3^{-n} .
$$

Given an integer $n \geqslant 2$ and $x \in \mathbf{R}$, set

$$
\psi_{n}(x)=n \delta_{n}^{-1} \varphi_{n}\left(x-2 \cdot 3^{-n}\right),
$$

where $\delta_{n}=\left(\int_{\mathbf{R}} \varphi_{n}^{4}(x) d x\right)^{1 / 2}$. Put $\psi(x)=\sum_{n=2}^{\infty} \psi_{n}(x)(x \in \mathbf{R})$. Clearly, supp $\psi \subset J$.

Let $L^{2}(\mathbf{R})$ be the Hilbert space of all (classes of) complex square integrable functions on $\mathbf{R}$, endowed with the norm $\|\cdot\|_{2}$.

Let $\pi_{\psi}$ be the multiplication operator defined by $\pi_{\psi} f=\psi f\left(f \in D\left(\pi_{\psi}\right)\right)$, with the domain $D\left(\pi_{\psi}\right)=\left\{f \in L^{2}(\mathbf{R}): \psi f \in L^{2}(\mathbf{R})\right\} . \pi_{\psi}$ is a selfadjoint operator in $L^{2}(\mathbf{R})$ and $A_{1}=i \pi_{\psi}$ is the generator of the unitary strongly continuous group $\left(e^{A_{1} t}\right)_{t \in \mathbf{R}}$ in $L^{2}(\mathbf{R})$ defined by

$$
\left(e^{A_{1} t} f\right)(x)=e^{i t \psi(x)} f(x) \quad\left(f \in L^{2}(\mathbf{R}), x, t \in \mathbf{R}\right) .
$$

Let $A_{2}$ be the generator of the unitary strongly continuous group $\left(e^{A_{2} t}\right)_{t \in \mathbf{R}}$ in $L^{2}(\mathbf{R})$ given by

$$
\left(e^{A_{2} t} f\right)(x)=f(x-t) \quad\left(f \in L^{2}(\mathbf{R}), x, t \in \mathbf{R}\right) .
$$


It is easily checked that for any $f \in C^{1}(\mathbf{R}) \cap L^{2}(\mathbf{R})$ with $f^{\prime} \in L^{2}(\mathbf{R}), f$ is in $D\left(A_{2}\right)$ and $A_{2} f=-f^{\prime}$.

Let $D_{0}$ be the set of all functions of the form

$$
x \rightarrow \varphi^{(k)}(x-u) \prod_{j=1}^{m} e^{i t_{j} \psi\left(x-s_{j}\right)} \quad\left(t_{j}, s_{j}, u \in \mathbf{R}, m, k \in \mathbf{N}\right) .
$$

Proposition 2.3. The following conditions are satisfied:

(1) $\left(e^{A_{1} t}\left(D_{0}\right) \cup e^{A_{2} t}\left(D_{0}\right)\right) \subset D_{0}$ for each $t \in \mathbf{R}$;

(2) $D_{0} \subset D\left(A_{1}\right)$ and $A_{1}\left(D_{0}\right) \subset C_{0}^{\infty}(\mathbf{R})$;

(3) $D_{0} \subset D\left(A_{2}\right)$ and, for each $f \in D_{0}, A_{2} f=g_{1}+g_{2}$ with $g_{1} \in D_{0}$ and $g_{2} \in$ $C_{0}^{\infty}(\mathbf{R})$.

Proof. (1) is evident.

(2) For any $f: x \rightarrow \varphi^{(k)}(x-u) \prod_{j=1}^{m} e^{i t ; \psi\left(x-s_{j}\right)} \quad\left(t_{j}, s_{j}, u \in \mathbf{R}, \quad m, k \in \mathbf{N}\right)$, the support of $\psi f$ is contained in $(I+u) \cap J$ and hence, by Proposition 2.2, is compact. Thus $f \in D\left(A_{1}\right)$ and $A_{1} f \in C_{0}^{\infty}(\mathbf{R})$.

(3) If $f \in D_{0}$ takes the form as in the paragraph above, then, since all derivatives of $\varphi$ are square integrable, $f$ is in $D\left(A_{2}\right)$ and $A_{2} f=g_{1}+g_{2}$, where

$$
\begin{array}{r}
g_{1}(x)=-\varphi^{(k+1)}(x-u) \prod_{j=1}^{m} e^{i t_{j} \psi\left(x-s_{j}\right)} \text { and } g_{2}(x)=-i f(x) \sum_{j=1}^{m} t_{j} \psi^{\prime}\left(x-s_{j}\right) \\
(x \in \mathbf{R}) .
\end{array}
$$

It is clear that $g_{1} \in D_{0}$. Since supp $g_{2} \subset(I+u) \cap \bigcup_{j=1}^{m}\left(J+s_{j}\right)$, it follows from Proposition 2.2 that $g_{2} \in C_{0}^{\infty}(\mathbf{R})$.

The proof is complete.

Let $D=\left\{f \in C^{\infty}(\mathbf{R}): f=f_{1}+f_{2}, f_{1} \in C_{0}^{\infty}(\mathbf{R}), f_{2} \in \operatorname{span} D_{0}\right\}$, where $\operatorname{span} D_{0}$ denotes the linear space spanned by $D_{0}$.

We now state our major result.

THEOREM 2.4. $D$ is a dense subspace of $L^{2}(\mathbf{R})$ contained in $D\left(A_{1}\right) \cap D\left(A_{2}\right)$ such that $\left(A_{1}(D) \cup A_{2}(D)\right) \subset D$ and $\left(e^{A_{1} t}(D) \cup e^{A_{2} t}(D)\right) \subset D$ for each $t \in \mathbf{R}$. Moreover, $\varphi$ is an element of $D$ such that the function $t \rightarrow\left\|A_{1} e^{A_{2} t} \varphi\right\|_{2}$ is bounded in no neighborhood of 0 .

Proof. In view of Proposition 2.3, only the last assertion requires a proof.

For each $n \in \mathbf{N}$ and each $x \in \mathbf{R}$, we have

$$
\left(A_{1} e^{2 \cdot 3^{-n} A_{2}} \varphi\right)(x)=i \sum_{k, l=2}^{\infty} \psi_{k}(x) \varphi_{l}\left(x-2 \cdot 3^{-n}\right) \text {. }
$$

Since $\psi_{n}(x) \varphi_{n}\left(x-2 \cdot 3^{-n}\right)=n \delta_{n}^{-1} \varphi_{n}^{2}\left(x-2 \cdot 3^{-n}\right) \neq 0$ for some $x \in \mathbf{R}$, it follows from Proposition 2.1 that if $k \neq n$ or $l \neq n$, then $\psi_{l}(x) \varphi_{k}\left(x-2 \cdot 3^{-n}\right)=0$ for all $x \in \mathbf{R}$. Consequently,

$$
\left\|A_{1} e^{2 \cdot 3^{n} A_{2}} \varphi\right\|_{2}=n \delta_{n}^{-1}\left(\int_{\mathbf{R}} \varphi_{n}^{4}\left(x-2 \cdot 3^{-n}\right) d x\right)^{1 / 2},
$$

from which the conclusion follows. 


\section{REFERENCES}

1. P. E. T. Jørgensen and R. T. Moore, Operator commutation relations, commutation relations for operators, semigroups, and resolvents with applications to mathematical physics and representations of Lie groups, Reidel, Dordrecht, 1984.

Mathematics Institute, University of Warsaw, PaŁaC Kultury i Nauki, IXP, 00-901 WarszaWa, POLAND 